\title{
Planar Double Box Integral for Top Pair Production with a Closed Top Loop to all orders in the Dimensional Regularization Parameter
}

\author{
Luise Adams, Ekta Chaubey, and Stefan Weinzierl \\ PRISMA Cluster of Excellence, Institut für Physik, Johannes Gutenberg-Universität Mainz, D-55099 Mainz, Germany
}

(Received 1 May 2018; published 1 October 2018)

\begin{abstract}
We compute systematically for the planar double box Feynman integral relevant to top pair production with a closed top loop the Laurent expansion in the dimensional regularization parameter $\varepsilon$. This is done by transforming the system of differential equations for this integral and all its sub-topologies to a form linear in $\varepsilon$, where the $\varepsilon^{0}$ part is strictly lower triangular. This system is easily solved order by order in the dimensional regularization parameter $\varepsilon$. This is an example of an elliptic multiscale integral involving several elliptic subtopologies. Our methods are applicable to similar problems.
\end{abstract}

DOI: 10.1103/PhysRevLett.121.142001

Introduction.-The physics of heavy elementary particles like the Higgs boson, the top quark, or the $W$ and $Z$ bosons plays an important role at the LHC and future colliders. Precision particle physics at these colliders relies crucially on our abilities to perform higher-order perturbative calculations and, in particular, on our abilities to compute the relevant Feynman integrals. The method of differential equations [1-9] has been used successfully for many Feynman integrals that evaluate to multiple polylogarithms [10-14]. For a large number of scattering processes with massless particles this is sufficient. However, as soon as massive particles enter the game, it is known that starting at two loops multiple polylogarithms will not be sufficient to express the Feynman integrals. The simplest example of a Feynman integral not expressible in terms of multiple polylogarithms is the two-loop sunrise integral with equal nonzero internal masses [15-34]. This integral is related to an elliptic curve and can be expressed to all orders in the dimensional regularization parameter $\varepsilon$ in iterated integrals of modular forms of $\Gamma_{1}(6)$. Integrals, which do not evaluate to multiple polylogarithms are now an active field of study in particle physics [35-52] and string theory [53-58].

In this Letter we report on a more involved computation. We consider the planar double box integral relevant to toppair production with a closed top loop. This integral enters the next-to-next-to-leading order (NNLO) contribution for the process $p p \rightarrow t \bar{t}$. Up to now, it is not known analytically. The existing NNLO calculation for this process uses numerical approximations for this integral $[59,60]$. Our inability to compute this integral analytically has been a

Published by the American Physical Society under the terms of the Creative Commons Attribution 4.0 International license. Further distribution of this work must maintain attribution to the author(s) and the published article's title, journal citation, and DOI. Funded by SCOAP ${ }^{3}$. show stopper for further progress on the analytical side. In this Letter we show how to compute analytically this integral. Our methods are applicable to similar problems.

The planar double box integral depends on two scales (e.g., $s / \mathrm{m}^{2}$ and $t / \mathrm{m}^{2}$, where $s$ and $t$ are the usual Mandelstam variables and $m$ the mass of the heavy particle). It involves the sunrise graph as a subtopology. Therefore, we do not expect this integral to evaluate to multiple polylogarithms. Phrased differently, we expect to see elliptic generalizations of multiple polylogarithms. An obvious question is: Which elliptic curve? To some surprise, there is not a single elliptic curve associated to this integral, but three different ones. We show in this Letter how to extract the elliptic curves from the maximal cuts of the (sub-) topologies. From these elliptic curves we obtain their periods.

In the next step we bring the system of differential equations to a form linear in $\varepsilon$, where the $\varepsilon^{0}$ part is strictly lower triangular. We introduce kinematic variables $x$ and $y$, which rationalize the square roots in the polylogarithmic case (i.e., for $t=m^{2}$ ). The transformation of the basis of master integrals is not rational in $x$ and $y$; however we find a transformation which is rational in $x, y$, the periods of the three elliptic curves, and their $y$ derivatives. Note that a system of differential equations linear in $\varepsilon$, where the $\varepsilon^{0}$ part is strictly lower triangular, can be easily transformed to an $\varepsilon$ form (i.e., without any $\varepsilon^{0}$ part) by introducing primitives for the terms occurring in the $\varepsilon^{0}$ part. Both systems are equivalent and both are easily solved order by order in the dimensional regularization parameter $\varepsilon$. For the case at hand the required primitives are usually transcendental functions. We prefer to work with a system linear in $\varepsilon$, where in the transformation matrix only the periods and their derivatives occur as transcendental functions.

There are two interesting cases, where the solution for the Feynman integrals simplify: for $t=m^{2}$ the solution degenerates to multiple polylogarithms, for $s=\infty$ the 


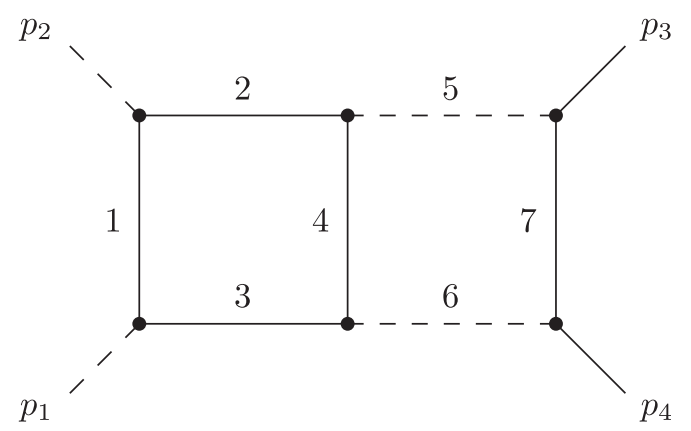

FIG. 1. The planar double box. Solid lines correspond to massive propagators of mass $m$, dashed lines correspond to massless propagators. All external momenta are out going and on shell: $p_{1}^{2}=p_{2}^{2}=0$ and $p_{3}^{2}=p_{4}^{2}=m^{2}$.

solution degenerates to iterated integrals of modular forms for $\Gamma_{1}(6)$.

The integral.-We consider the planar double box integral shown in Fig. 1. This integral is relevant to the NNLO corrections for $t \bar{t}$ production at the LHC. In Fig. 1 the solid lines correspond to propagators with a mass $m$, while dashed lines correspond to massless propagators. All external momenta are out going and on shell. The Mandelstam variables are defined as usual,

$$
s=\left(p_{1}+p_{2}\right)^{2}, \quad t=\left(p_{2}+p_{3}\right)^{2} .
$$

We are interested in the dimensional regulated integral

$$
\begin{aligned}
I_{\nu_{1} \nu_{2} \nu_{3} \nu_{4} \nu_{5} \nu_{6} \nu_{7}}\left(D, \frac{s}{m^{2}}, \frac{t}{m^{2}}\right)= & e^{2 \gamma_{E} \varepsilon}\left(m^{2}\right)^{\sum_{j=1}^{7} \nu_{j}-D} \\
& \times \int \frac{d^{D} k_{1}}{i \pi^{\frac{D}{2}}} \frac{d^{D} k_{2}}{i \pi^{\frac{D}{2}}} \prod_{j=1}^{7} \frac{1}{P_{j}^{\nu_{j}}},
\end{aligned}
$$

where $\gamma_{E}$ denotes the Euler-Mascheroni constant, $D=$ $4-2 \varepsilon$ denotes the dimension of space-time, and the propagators are given by

$P_{1}=-\left(k_{1}+p_{2}\right)^{2}+m^{2}, \quad P_{2}=-k_{1}^{2}+m^{2}$,

$P_{3}=-\left(k_{1}+p_{1}+p_{2}\right)^{2}+m^{2}, \quad P_{4}=-\left(k_{1}+k_{2}\right)^{2}+m^{2}$,

$P_{5}=-k_{2}^{2}, \quad P_{6}=-\left(k_{2}+p_{3}+p_{4}\right)^{2}$,

$P_{7}=-\left(k_{2}+p_{3}\right)^{2}+m^{2}$.

This integral has a Laurent expansion in $\varepsilon$ :

$$
I_{\nu_{1} \nu_{2} \nu_{3} \nu_{4} \nu_{5} \nu_{6} \nu_{7}}=\sum_{j=j_{\min }}^{\infty} \varepsilon^{j} I_{\nu_{1} \nu_{2} \nu_{3} \nu_{4} \nu_{5} \nu_{6} \nu_{7}}^{(j)}
$$

In this Letter we present a method to systematically compute the $j$ th term of the $\varepsilon$ expansion. The result is expressed in terms of iterated integrals [61]. If $\omega_{1}, \ldots, \omega_{k}$ are differential one forms on a manifold $M$ and $\gamma:[0,1] \rightarrow M$ a path, we

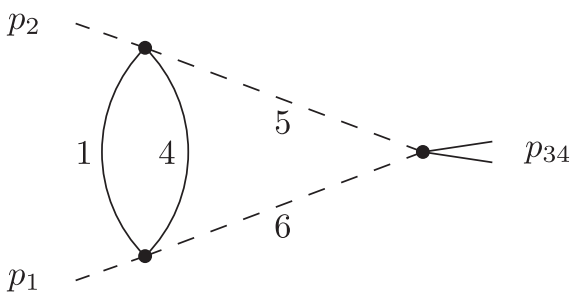

FIG. 2. A subtopology leading to the square root $\sqrt{-s\left(-4 m^{2}-s\right)}$.

write for the pull back of $\omega_{j}$ to the interval $[0,1]$,

$$
f_{j}(\lambda) d \lambda=\gamma^{*} \omega_{j}
$$

The iterated integral is then defined by

$$
\begin{gathered}
I_{\gamma}\left(\omega_{1}, \ldots, \omega_{k} ; \lambda\right)= \\
\int_{0}^{\lambda} d \lambda_{1} f_{1}\left(\lambda_{1}\right) \int_{0}^{\lambda_{1}} d \lambda_{2} f_{2}\left(\lambda_{2}\right) \ldots \int_{0}^{\lambda_{k-1}} d \lambda_{k} f_{k}\left(\lambda_{k}\right) .
\end{gathered}
$$

Multiple polylogarithms are iterated integrals, where all differential one forms are of the form

$$
\omega_{j}=\frac{d \lambda}{\lambda-c_{j}} .
$$

If $f(\tau)$ is a modular form, we simply write with a slight abuse of notation $f$ instead of $2 \pi i f d \tau$ in the arguments of iterated integrals.

The kinematic variables for the multiple polylogarithms. - The Feynman integral is a function of two kinematic ratios, say $s / m^{2}$ and $t / m^{2}$. A significant fraction of the subtopologies depends only on $s / \mathrm{m}^{2}$, but not on $t / \mathrm{m}^{2}$. These integrals are expressible in terms of multiple polylogarithms and their system of differential equations can be transformed to an $\varepsilon$ form. This introduces square roots, which are absorbed by a change of kinematic variables. The square root $\sqrt{-s\left(4 m^{2}-s\right)}$ is typical for massive Feynman integrals; however there are also subtopologies, which lead to the square root $\sqrt{-s\left(-4 m^{2}-s\right)}$ (note the sign in front of $4 \mathrm{~m}^{2}$ ). An example is shown in Fig. 2. A transformation, which absorbs both square roots simultaneously is given by

$$
\frac{s}{m^{2}}=-\frac{\left(1+x^{2}\right)^{2}}{x\left(1-x^{2}\right)}, \quad \frac{t}{m^{2}}=y .
$$

This defines the variables $x$ and $y$. The variable $y$ is not needed for integrals depending only on $s / \mathrm{m}^{2}$. For the integrals depending only on $s / \mathrm{m}^{2}$ we introduce five differential one forms 


$$
\begin{aligned}
\omega_{0}= & \frac{d s}{s}=\frac{2 d x}{x-i}+\frac{2 d x}{x+i}-\frac{d x}{x-1}-\frac{d x}{x+1}-\frac{d x}{x}, \\
\omega_{4}= & \frac{d s}{s-4 m^{2}}=\frac{2 d x}{x-(1+\sqrt{2})}+\frac{2 d x}{x-(1-\sqrt{2})} \\
& -\frac{d x}{x-1}-\frac{d x}{x+1}-\frac{d x}{x}, \\
\omega_{-4}= & \frac{d s}{s+4 m^{2}}=\frac{2 d x}{x-(-1+\sqrt{2})}+\frac{2 d x}{x-(-1-\sqrt{2})} \\
& -\frac{d x}{x-1}-\frac{d x}{x+1}-\frac{d x}{x}, \\
\omega_{0,4}= & \frac{d s}{\sqrt{-s\left(4 m^{2}-s\right)}}=\frac{d x}{x-1}-\frac{d x}{x+1}+\frac{d x}{x}, \\
\omega_{-4,0}= & \frac{d s}{\sqrt{-s\left(-4 m^{2}-s\right)}}=-\frac{d x}{x-1}+\frac{d x}{x+1}+\frac{d x}{x} .
\end{aligned}
$$

Then all subtopologies, which depend only on $s / \mathrm{m}^{2}$, can be expressed as iterated integrals with letters given by these five differential one forms. From Eq. (9) it is clear that they are expressible in terms of multiple polylogarithms.

Elliptic curves.-Let us consider an elliptic curve defined by the quartic equation

$$
E: w^{2}=\left(z-z_{1}\right)\left(z-z_{2}\right)\left(z-z_{3}\right)\left(z-z_{4}\right) .
$$

We set

$$
\begin{aligned}
& Z_{1}=\left(z_{2}-z_{1}\right)\left(z_{4}-z_{3}\right), \\
& Z_{2}=\left(z_{3}-z_{2}\right)\left(z_{4}-z_{1}\right), \\
& Z_{3}=\left(z_{3}-z_{1}\right)\left(z_{4}-z_{2}\right),
\end{aligned}
$$

and define the modulus and the complementary modulus

$$
k^{2}=\frac{Z_{1}}{Z_{3}}, \quad \bar{k}^{2}=\frac{Z_{2}}{Z_{3}} .
$$

Our standard choice for the periods is

$$
\psi_{1}=\frac{4 K(k)}{Z_{3}^{\frac{1}{2}}}, \quad \psi_{2}=\frac{4 i K(\bar{k})}{Z_{3}^{\frac{1}{2}}},
$$

where $K(x)$ denotes the complete elliptic integral of the first kind. For the double box integral we have to consider three elliptic curves $E^{(a)}, E^{(b)}$, and $E^{(c)}$, which occur for the first time in the three Feynman graphs shown in Fig. 3. The equations of the elliptic curves are extracted from the maximal cuts of these Feynman integrals [62-69], specifically from the maximal cuts of

$$
I_{1001001}(2-2 \varepsilon), \quad I_{1112001}(4-2 \varepsilon), \quad I_{2001111}(4-2 \varepsilon) .
$$

For these three integrals, the elliptic curves are most easily obtained from the loop-by-loop approach in the Baikov representation [67]. We find for all three curves
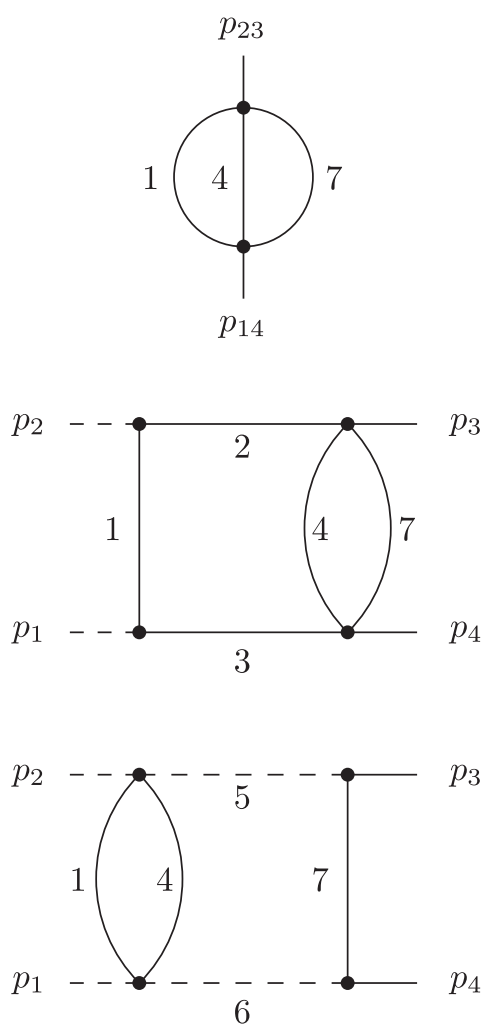

FIG. 3. The Feynman graphs associated to the three elliptic curves.

$$
z_{1}^{(a, b, c)}=\frac{t}{m^{2}}-4, \quad z_{4}^{(a, b, c)}=\frac{t}{m^{2}} .
$$

They differ in the values for the roots $z_{2}$ and $z_{3}$. We have

$z_{2,3}^{(a)}=-1 \mp 2 \sqrt{\frac{t}{m}}$,

$z_{2,3}^{(b)}=-1 \mp 2 \sqrt{\frac{t}{m^{2}}+\frac{\left(m^{2}-t\right)^{2}}{m^{2} s}}$,

$z_{2,3}^{(c)}=-\frac{(s+4 t)}{\left(s-4 m^{2}\right)} \mp \frac{2}{4 m^{2}-s} \sqrt{\frac{s}{m^{2}}\left(s t+\left(m^{2}-t\right)^{2}\right)}$.

It is easily checked by computing the $j$ invariants that the three curves are not isomorphic. However, the curves $E^{(b)}$ and $E^{(c)}$ degenerate to curve $E^{(a)}$ in the limit $s \rightarrow \infty$. Associated to the curve $E^{(a)}$ are modular forms of $\Gamma_{1}(6)$. We set

$$
\begin{aligned}
& g_{n, r}=-\frac{1}{2} \frac{y(y-1)(y-9)}{y-r}\left(\frac{\psi_{1}^{(a)}}{\pi}\right)^{n}, \\
& p_{n, s}=-\frac{1}{2} y(y-1)^{1+s}(y-9)\left(\frac{\psi_{1}^{(a)}}{\pi}\right)^{n},
\end{aligned}
$$




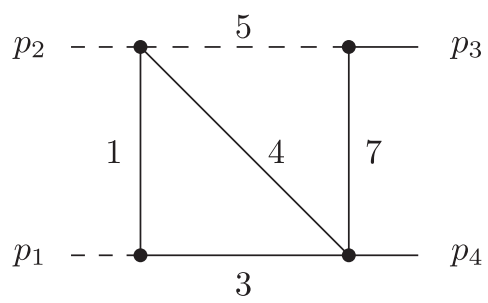

FIG. 4. A subtopology with an additional relation.

Relevant to the problem is the set

$$
\left\{1, g_{2,0}, g_{2,1}, g_{2,9}, g_{3,1}, p_{3,0}, g_{4,0}, g_{4,1}, g_{4,9}, p_{4,0}, p_{4,1}\right\} \text {. }
$$

These are modular forms of $\Gamma_{1}(6)$ in the variable $\tau_{6}=$ $\psi_{2}^{(a)} /\left(6 \psi_{1}^{(a)}\right)$, which we may substitute for the variable $y$.

Master integrals and differential equations.-In order to derive the system of differential equations we first used ReduZE [70], KIRA [71], and Fire [72] for the integral reductions. Taking trivial symmetry relations into account, all programs give 45 master integrals. However, the reductions disagree for the three most complicated topologies. For a given set of master integrals $\vec{I}$ we obtain the system of differential equations

$$
d \vec{I}=A(\varepsilon, x, y) \vec{I}
$$

In general, this system is not yet linear in $\varepsilon$, but it should satisfy the integrability condition

$$
d A=A \wedge A .
$$

At first sight, the results of two of three programs above fail the integrability check. Still, all three programs correctly implement the Laporta algorithm [73]. However, the Laporta algorithm does not guarantee that all relations among the Feynman integrals are found. Here, we have an example where one additional relation exists in the subtopology shown in Fig. 4. This additional relation reduces the number of master integrals in this topology from 5 to 4 . Imposing this relation, the results from REDUZE, KIRA, and FIRE agree and the integrability condition is satisfied. In addition, we verified numerically the first few terms in the $\varepsilon$ expansion of this relation. The extra relation comes from an IBP identity of a higher sector (i.e., the topology $I_{\nu_{1} \nu_{2} 0 \nu_{4} \nu_{5} \nu_{6} \nu_{7}}$ ), where the coefficients of the integrals of the higher sector are 0 . We would like to add that ReDuzE is able to find the relation and can be forced to use this relation with the command distribute_external [74].

In this Letter we are interested in the integral $I_{1111111}$. With the help of the methods from Ref. [9] we may decouple two integrals in the top topology. Thus we have to consider a system of 42 master integrals for $I_{1111111}$.
Under a change of basis

$$
\vec{J}=U \vec{I},
$$

the differential equations transform into

$$
d \vec{J}=A^{\prime} \vec{J}, \quad A^{\prime}=U A U^{-1}+U d U^{-1} .
$$

The main result of this Letter is that there exists a transformation $U$, such that

$$
d \vec{J}=\left(A^{(0)}(x, y)+\varepsilon A^{(1)}(x, y)\right) \vec{J},
$$

and $A^{(0)}$ is strictly lower triangular (i.e., $A_{i j}^{(0)}=0$ for $j \geq i$ ). The system of differential equations is linear in $\varepsilon$ and easily solved order by order in $\varepsilon$ in terms of iterated integrals. The transformation matrix is rational in

$$
\varepsilon, x, y, \psi_{1}^{(a)}, \psi_{1}^{(b)}, \psi_{1}^{(c)}, \partial_{y} \psi_{1}^{(a)}, \partial_{y} \psi_{1}^{(b)}, \partial_{y} \psi_{1}^{(c)} .
$$

We constructed this matrix by analyzing the Picard-Fuchs operators in the diagonal blocks [9] and by using a slightly modified version of the algorithm of Meyer $[75,76]$ for the nondiagonal blocks. To give an example, the three master integrals in the topology $I_{\nu_{1} \nu_{2} \nu_{3} \nu_{4} 00 \nu_{7}}$ can be taken as

$$
\begin{aligned}
J_{24}= & \varepsilon^{3} \frac{\left(1+x^{2}\right)^{2}}{x\left(1-x^{2}\right)} \frac{\pi}{\psi_{1}^{(b)}} I_{1112001}, \\
J_{25}= & \varepsilon^{3}(1-2 \varepsilon) \frac{\left(1+x^{2}\right)^{2}}{x\left(1-x^{2}\right)} I_{1111001}+R_{25,24} \frac{\psi_{1}^{(b)}}{\pi} J_{24}, \\
J_{26}= & \frac{6}{\varepsilon} \frac{\left(\psi_{1}^{(b)}\right)^{2}}{2 \pi i W_{y}^{(b)}} \frac{d}{d y} J_{24}+R_{26,24}\left(\frac{\psi_{1}^{(b)}}{\pi}\right)^{2} J_{24} \\
& -\frac{\varepsilon^{2}}{24}\left(y^{2}-30 y-27\right) \frac{\psi_{1}^{(b)}}{\pi} \mathbf{D}^{-} I_{1001001},
\end{aligned}
$$

where $R_{25,24}$ and $R_{26,24}$ are rational functions in $(x, y), \mathbf{D}^{-}$ denotes the dimension shift operator $D \rightarrow D-2$, and $W_{y}^{(b)}$ the Wronskian

$$
W_{y}^{(b)}=\psi_{1}^{(b)} \partial_{y} \psi_{2}^{(b)}-\psi_{2}^{(b)} \partial_{y} \psi_{1}^{(b)} .
$$

As in the sunrise sector [40], one integral is divided by a period $\left(J_{24}\right)$, while a second integral is given as a derivative plus additional terms $\left(J_{26}\right)$. This pattern applies to all elliptic sectors.

The matrix $A^{(0)}$ in Eq. (22) vanishes for $x=0$ or $y=1$. The occurrence of $\varepsilon^{0}$ terms in the differential equations is expected from the study of the sunrise integral with unequal masses [29,33]. For $y=1$ the entries of $A^{(1)}$ reduce to the differential one forms of Eq. (9), for $x=0$ they reduce to the modular forms of Eq. (17). The solution reduces 
therefore to multiple polylogarithms for $y=1$ and to iterated integrals of modular forms for $x=0$. We have compared numerically the solutions of all master integrals with results from sector_decomposition [77] and found agreement. Albeit the transformation $U$ significantly simplifies the system of differential equations, the length of the solution still exceeds the format of this Letter. The definition of the master integrals, the differential equation, and the results are given in the Supplemental Material [78]. In addition, a longer publication [79] describes the details of our calculation.

Conclusions. - In this Letter we analyzed the planar double box integral relevant to top pair production with a closed top loop. This integral depends on two scales and involves several elliptic subsectors. This integral has not been known analytically and impedes further progress on the analytic computation of higher-loop Feynman integrals with massive particles. In this Letter we reported that we may transform the system of differential equations to a form linear in $\varepsilon$, where the $\varepsilon^{0}$ term is strictly lower triangular. With such a linear form the solution in terms of iterated integrals is immediate. Our techniques open the door for more complicated Feynman integrals.

L. A. and E. C. are grateful for financial support from the research training group Graduiertenkolleg 1581. S.W. would like to thank the Hausdorff Research Institute for Mathematics for hospitality, where part of this work was carried out.

[1] A. V. Kotikov, Phys. Lett. B 254, 158 (1991).

[2] A. V. Kotikov, Phys. Lett. B 267, 123 (1991).

[3] E. Remiddi, Nuovo Cim. A 110, 1435 (1997).

[4] T. Gehrmann and E. Remiddi, Nucl. Phys. B580, 485 (2000).

[5] M. Argeri and P. Mastrolia, Int. J. Mod. Phys. A 22, 4375 (2007).

[6] S. Müller-Stach, S. Weinzierl, and R. Zayadeh, Commun. Math. Phys. 326, 237 (2014).

[7] J. M. Henn, Phys. Rev. Lett. 110, 251601 (2013).

[8] J. M. Henn, J. Phys. A 48, 153001 (2015).

[9] L. Adams, E. Chaubey, and S. Weinzierl, Phys. Rev. Lett. 118, 141602 (2017)

[10] A. B. Goncharov, Math. Res. Lett. 5, 497 (1998).

[11] A. B. Goncharov, arXiv:math/0103059.

[12] J. M. Borwein, D. M. Bradley, D. J. Broadhurst, and P. Lisonek, Trans. Am. Math. Soc. 353, 907 (2001).

[13] S. Moch, P. Uwer, and S. Weinzierl, J. Math. Phys. (N.Y.) 43, 3363 (2002).

[14] J. Vollinga and S. Weinzierl, Comput. Phys. Commun. 167, 177 (2005).

[15] D. J. Broadhurst, J. Fleischer, and O. Tarasov, Z. Phys. C 60, 287 (1993).

[16] F. A. Berends, M. Buza, M. Böhm, and R. Scharf, Z. Phys. C 63, 227 (1994).

[17] S. Bauberger, M. Böhm, G. Weiglein, F. A. Berends, and M. Buza, Nucl. Phys. B 37, 95 (1994).
[18] S. Bauberger, F. A. Berends, M. Böhm, and M. Buza, Nucl. Phys. B434, 383 (1995).

[19] S. Bauberger and M. Böhm, Nucl. Phys. B445, 25 (1995).

[20] M. Caffo, H. Czyz, S. Laporta, and E. Remiddi, Nuovo Cim. A 111, 365 (1998).

[21] S. Laporta and E. Remiddi, Nucl. Phys. B704, 349 (2005).

[22] B. A. Kniehl, A. V. Kotikov, A. Onishchenko, and O. Veretin, Nucl. Phys. B738, 306 (2006).

[23] S. Groote, J. G. Körner, and A. A. Pivovarov, Ann. Phys. (Amsterdam) 322, 2374 (2007).

[24] S. Groote, J. Körner, and A. Pivovarov, Eur. Phys. J. C 72, 2085 (2012).

[25] D. H. Bailey, J. M. Borwein, D. Broadhurst, and M. L. Glasser, J. Phys. A 41, 205203 (2008).

[26] S. Müller-Stach, S. Weinzierl, and R. Zayadeh, Commun. Theor. Phys. 6, 203 (2012).

[27] L. Adams, C. Bogner, and S. Weinzierl, J. Math. Phys. (N.Y.) 54, 052303 (2013).

[28] S. Bloch and P. Vanhove, J. Number Theory 148, 328 (2015).

[29] L. Adams, C. Bogner, and S. Weinzierl, J. Math. Phys. (N.Y.) 55, 102301 (2014).

[30] L. Adams, C. Bogner, and S. Weinzierl, J. Math. Phys. (N.Y.) 56, 072303 (2015).

[31] L. Adams, C. Bogner, and S. Weinzierl, J. Math. Phys. (N.Y.) 57, 032304 (2016).

[32] E. Remiddi and L. Tancredi, Nucl. Phys. B880, 343 (2014).

[33] S. Bloch, M. Kerr, and P. Vanhove, Adv. Theor. Math. Phys. 21, 1373 (2017).

[34] S. Groote and J. G. Körner, arXiv:1804.10570.

[35] S. Bloch, M. Kerr, and P. Vanhove, Compos. Math. 151, 2329 (2015).

[36] E. Remiddi and L. Tancredi, Nucl. Phys. B907, 400 (2016).

[37] L. Adams, C. Bogner, A. Schweitzer, and S. Weinzierl, J. Math. Phys. (N.Y.) 57, 122302 (2016).

[38] L. Adams and S. Weinzierl, Commun. Theor. Phys. 12, 193 (2018).

[39] C. Bogner, A. Schweitzer, and S. Weinzierl, Nucl. Phys. B922, 528 (2017).

[40] L. Adams and S. Weinzierl, Phys. Lett. B 781, 270 (2018).

[41] M. Sø gaard and Y. Zhang, Phys. Rev. D 91, 081701 (2015).

[42] R. Bonciani, V. Del Duca, H. Frellesvig, J. M. Henn, F. Moriello, and V. A. Smirnov, J. High Energy Phys. 12 (2016) 096.

[43] A. von Manteuffel and L. Tancredi, J. High Energy Phys. 06 (2017) 127.

[44] A. Primo and L. Tancredi, Nucl. Phys. B921, 316 (2017).

[45] J. Ablinger, J. Blümlein, A. De Freitas, M. van Hoeij, E. Imamoglu, C. G. Raab, C.-S. Radu, and C. Schneider, J. Math. Phys. (N.Y.) 59, 062305 (2018).

[46] J. L. Bourjaily, A. J. McLeod, M. Spradlin, M. von Hippel, and M. Wilhelm, Phys. Rev. Lett. 120, 121603 (2018).

[47] M. Hidding and F. Moriello, arXiv:1712.04441.

[48] G. Passarino, Eur. Phys. J. C 77, 77 (2017).

[49] E. Remiddi and L. Tancredi, Nucl. Phys. B925, 212 (2017).

[50] J. Broedel, C. Duhr, F. Dulat, and L. Tancredi, J. High Energy Phys. 05 (2018) 093.

[51] J. Broedel, C. Duhr, F. Dulat, and L. Tancredi, Phys. Rev. D 97, 116009 (2018). 
[52] J. Broedel, C. Duhr, F. Dulat, B. Penante, and L. Tancredi, J. High Energy Phys. 08 (2018) 014.

[53] J. Broedel, C. R. Mafra, N. Matthes, and O. Schlotterer, J. High Energy Phys. 07 (2015) 112.

[54] J. Broedel, N. Matthes, and O. Schlotterer, J. Phys. A 49, 155203 (2016).

[55] J. Broedel, N. Matthes, G. Richter, and O. Schlotterer, J. Phys. A 51, 285401 (2018).

[56] E. D’Hoker, M. B. Green, Ö. Gürdogan, and P. Vanhove, Commun. Theor. Phys. 11, 165 (2017).

[57] S. Hohenegger and S. Stieberger, Nucl. Phys. B925, 63 (2017).

[58] J. Broedel, O. Schlotterer, and F. Zerbini, arXiv:1803.00527.

[59] M. Czakon, P. Fiedler, and A. Mitov, Phys. Rev. Lett. 110, 252004 (2013).

[60] P. Bärnreuther, M. Czakon, and P. Fiedler, J. High Energy Phys. 02 (2014) 078.

[61] K.-T. Chen, Bull. Am. Math. Soc. 83, 831 (1977).

[62] P. A. Baikov, Nucl. Instrum. Methods Phys. Res., Sect. A 389, 347 (1997).

[63] R. N. Lee, Nucl. Phys. B830, 474 (2010).

[64] D. A. Kosower and K. J. Larsen, Phys. Rev. D 85, 045017 (2012).

[65] S. Caron-Huot and K. J. Larsen, J. High Energy Phys. 10 (2012) 026.
[66] A. Primo and L. Tancredi, Nucl. Phys. B916, 94 (2017).

[67] H. Frellesvig and C. G. Papadopoulos, J. High Energy Phys. 04 (2017) 083.

[68] J. Bosma, M. Sogaard, and Y. Zhang, J. High Energy Phys. 08 (2017) 051.

[69] M. Harley, F. Moriello, and R. M. Schabinger, J. High Energy Phys. 06 (2017) 049.

[70] A. von Manteuffel and C. Studerus, arXiv:1201.4330.

[71] P. Maierhoefer, J. Usovitsch, and P. Uwer, Comput. Phys. Commun. 230, 99 (2018).

[72] A. V. Smirnov, Comput. Phys. Commun. 189, 182 (2015).

[73] S. Laporta, Int. J. Mod. Phys. A 15, 5087 (2000).

[74] We thank L. Tancredi for pointing this out and A. von Manteuffel for advice on Reduze.

[75] C. Meyer, J. High Energy Phys. 04 (2017) 006.

[76] C. Meyer, Comput. Phys. Commun. 222, 295 (2018).

[77] C. Bogner and S. Weinzierl, Comput. Phys. Commun. 178, 596 (2008).

[78] See Supplemental Material at http://link.aps.org/ supplemental/10.1103/PhysRevLett.121.142001 for the definition of the master integrals, the differential equation and the results.

[79] L. Adams, E. Chaubey, and S. Weinzierl, arXiv:1806.04981. 